\title{
Stigma in COVID-19: A barrier to seek medical care and family support
}

\author{
Mehran Asadi-Aliabadi ${ }^{1,2}$, Arash Tehrani-Banihashemi*1,3 $\mathbb{D}$, Maziar Moradi-Lakeh ${ }^{1,3}$ \\ Received: 21 May 2020 \\ Published: 13 Aug 2020
}

\begin{abstract}
The new coronavirus pandemic at the end of 2019 raised fear around the world. The rapid spread and relatively higher observed case fatality of this disease compared to other viral respiratory infections, have caused social reactions such as xenophobia and stigma. These negative social attitudes and acts will affect different segments of the society, such as patients and their families and health care providers. Also, this could disrupt the identification and surveillance of patients and could lead to considerable negative impacts on its control and management.
\end{abstract}

Keywords: Social Stigma, COVID-19, Health care providers

Conflicts of Interest: None declared

Funding: None

*This work has been published under CC BY-NC-SA 1.0 license.

Copyright $\odot$ Iran University of Medical Sciences

Cite this article as: Asadi-Aliabadi M, Tehrani-Banihashemi A, Moradi-Lakeh M. Stigma in COVID-19: A barrier to seek medical care and family support. Med J Islam Repub Iran. 2020 (13 Aug);34:98. https://doi.org/10.47176/mjiri.34.98

At the end of 2019, a novel infectious disease called Coronavirus 2019 (COVID-19) became the most important public health concern (1). Many efforts have been made to implement preventive behavior, treatment, and control this pandemic. The high infection and relatively high mortality rates of COVID-19 have caused global concern (1). Also, the fear of contact with people who may be infected with COVID-19 is mentioned in scientific evidence (2). Thus, the COVID-19 pandemic is highly stressful for people and communities.

We learned from previous infectious disease epidemics, such as HIV, SARS, and Ebola, that high infectivity and mortality with unknown natural history of a disease, along with the lack of awareness and false perception, the nature of COVID-19, and the lack of drugs and vaccines for treatment and prevention(3), will cause fear and anxiety in the society. However, stigma related to infectious diseases may have different causes. For example, HIV-related

Corresponding author: Dr Arash Tehrani-Banihashemi, tehrani.a@iums.ac.ir

1. Preventive Medicine and Public Health Research Center, Psychosocial Health Research Institute, Iran University of Medical Sciences, Tehran, Iran

2. Student Research Committee, Iran University of Medical Sciences, Tehran, Iran

3. Community and Family Medicine Department, School of Medicine, Iran University of Medical Sciences, Tehran, Iran stigma may be due to people's judgment about the common modes of transmission, such as sexual behaviors, shared needles, or syringe use in drug users. This prejudice, along with the incurable, chronic, and progressive course of the disease, puts HIV among the diseases with the highest stigma (4).

Fear of a disease can lead to social stigma toward people, places, etc. that are related to the disease. Social stigma in the field of health refers to a negative relationship between a person and a group of people who have certain characteristics or a specific disease (5). This can negatively affect people with the disease, as well as their families, friends, and communities (5). Therefore, social stigma can distort the public's perception of risk and deteriorate epidemic situation.

Behavioral manifestation of stigma is discrimination, which includes actions that could lead to lack of acceptance in the community and reduction in treatment

$\uparrow$ What is "already known" in this topic:

Following the rapid rise in morbidity and contagiousness of COVID-19, the related fear and psychological consequences are increasing in the community. Hence, reduction in the number of demands for diagnostic and treatment services due to fear of stigma will cause irreparable consequences.

$\rightarrow$ What this article adds:

In this study, the causes of stigma related to COVID-19 and subsequent strategies to reduce and control stigma are presented. 
opportunities and disease control (6). Discrimination has different social, physical, and mental health consequences. Prolonged and ongoing stigma in the society may cause the patients to internalize the stigma to themselves and reinforce self-discrimination due to shame and negative self-judgment. These negative feelings can affect the decision of individuals suspected to have the disease for getting tested and patients for seeking treatment services, which is very dangerous both at individual and community level with respect to disease control.

In some parts of the world some discriminatory social behaviors have been reported against people of a particular ethnicity who have been infected with COVID-19 (7).

Stigma can also cause anger in some people, which endangers the health and safety of others (8). For example, people with HIV have reported to experience rejection, anger, and avoidance (9). These factors can lead to feelings of shame and ridicule in these people (9). As a result, all these factors can lead to a hidden disease. Sources of anger in people living with HIV are as follow (9):

Health care professionals: Lack of services, such as dentistry, maternity hospital, laboratory, hospital services, a other health services; and insulting behavior towards these patients;

- Employers: Dismissal, nonemployment, or very difficult circumstances for patients;

- Family: Lack of respect for patients' privacy in the family leads to their identification by neighbors and relatives, and as a result they move away.

In addition to the psychological complications, there will be physical problems for those who have been stigmatized. Hence, stigmatized people in the community may be at risk for the following reasons (10):

- Marginalizing or social exclusion;

- Rejection of health care, education, housing, or employment;

- Physical or mental violence and harassment;

Different individuals and groups may be at risk of stigma due to COVID-19:

- People with a history of travel, especially to high-risk areas (11);

- Inhabitants of specific geographic regions, such as a specific country or a specific subnational region within a country (8);

- Infected people who have been fully recovered (11);

- People who have alarming symptoms and signs such as fever or cough;

- Health care providers (11), especially those who are directly involved with care for patients with COVID-19 and unofficial caregivers of patients;

- People at higher risk of more severe forms of COVID19, such as elder population (12) and people with chronic conditions (3); some people carelessly think these groups are responsible for the socioeconomic impact imposed by the social distance interventions and that others should not be limited because of the vulnerability of a specific group.

\section{Factors affecting the stigma control}

First, insufficient knowledge and awareness about transmission, treatment, and prevention of COVID-19 can increase the stigma in individuals and the society. Therefore, by providing accurate and timely information to the community about the affected areas, high-risk people, preventive measures, treatment suggestions, announcements of designated centers to provide relevant services, using simple language and nonuse of medical words in providing knowledge and awareness, we can take effective action to reduce fear and stigma. For implementation, social media is one of the main ways to reach a large number of people at a relatively low cost to provide this information (5).

Second, using reference groups, such as religious leaders, artists, sports heroes, and other celebrities, who are socially acceptable can be helpful in conveying health messages, supporting people who have been stigmatized, and finding ways to reduce it (5). Self-disclosure of COVID-19 infection by such people or sympathy of the reference groups show the Coronavirus does not target specific groups of people in terms of socioeconomic status, job, ethnicity, geographic region or nationality, which could reduce the stigma and discrimination.

Third, sharing success stories from local people in different regions that have experienced COVID-19 disease and been recovered or those who have contributed to their recovery, such as health care providers, can be effective to emphasize most people recover from COVID-19. Also, appreciating the role of health care providers, such as establishing a "National Health Heroes" campaign to honor health care providers may reduce stigma against health care providers (5).

Forth, we should ensure different ethnic groups have been portrayed in the reports. According to the available documents, this disease has affected most nations, races, religions of all genders and ages, regardless of economic and social status, and contribution of all members of societies are necessary to control it (5).

Fifth, Journalistic reporting that focuses too much on the responsibility of patients in spreading the virus in the community can stigmatize the patients. Therefore, there should be a balance between "individual behaviors and patients' responsibility for having and spreading COVID19 " and environmental factors that are not controlled by a single individual (13).

Sixth, emphasis on finding a vaccine and treatment to control this pandemic can increase fear and make us think we cannot stop infections now. Instead, we can promote content about the main methods of infection prevention, COVID-19 symptoms, and when to seek medical care (5).

While "social distancing" is highly recommended for controlling COVID-19, it can convey the wrong message and lead to social isolation and marginalization. Therefore, it is better to use physical distance. Also, travel restrictions may be a factor in stigmatizing and it can be caused by fear of the patient or the carrier of the virus (14).

Now, besides the campaigns suggested above, starting an "Antistigma" campaign might be effective, but it must 
continue after the end of this pandemic (15). It seems long-term effects on people who tested positive can be a lasting legacy of infection around the world. Under fear and confusion, the stigma will remain as a lasting stain on people who have been exposed to the disease in various forms. Hence, stigma and fear can severely hamper efforts to control COVID-19.

People and media should be careful not to use stigmatizing language and should correct the rumors, myths, and misinformation. Calling or addressing patients with negative Corona-related adjectives or linking the disease to a particular geographic region or ethnic group or gender should be prohibited and condemned (16).

Thus, increasing people's trust is very important, and this also depends on providing the right information about the current situation by the country's health officials in the media (7). Separating reality from rumor is highly important in this situation. If the facts are not told to people, superstitions and lies will take their place. Therefore, when communicating on social media and other communication platforms, it is better to behave responsibly and sensibly by showing supportive behaviors about COVID19 disease. On the other hand, we need to be cautious and instead of using the term social distancing use physical distancing to signify that maintaining a physical distance from others does not mean social isolation (17). Last but not least, the NGOs can help to better implement stigma reduction strategies (18).

\section{Conclusion}

Stigma can not only harm the patients and their relatives, but also it can be a barrier for effective control of this pandemic. Therefore, governments, citizens, the media, key individuals, and communities have an important role to play in preventing and stopping the stigma of COVID-19. Everyone can prevent COVID-19 stigma by understanding the facts and sharing them with others in their community.

\section{Conflict of Interests}

The authors declare that they have no competing interests.

\section{References}

1. Lin CY. Social reaction toward the 2019 novel coronavirus (COVID19). J Health Soc Behav. 2020;3(1):1.

2. Lum LHW, Tambyah PA. Outbreak of COVID-19-an urgent need for good science to silence our fears? Singap Med J. 2020;61(2):55.

3. Ryan DH, Ravussin E, Heymsfield S. COVID 19 and the Patient with Obesity-The Editors Speak Out. Obesity. 2020.

4. Davtyan M, Brown B, Folayan M. Addressing Ebola-related stigma: lessons learned from HIV/AIDS. Glob Health Action. 2014;7:26058.

5. Unicef. Social Stigma associated with COVID-19 2020 [Available from: https://www.unicef.org/documents/social-stigma-associated-coro navirus-disease-covid-19.

6. Stangl AL, Earnshaw VA, Logie CH, van Brakel W, Simbayi LC, Barré I, et al. The Health Stigma and Discrimination Framework: a global, crosscutting framework to inform research, intervention development, and policy on health-related stigmas. BMC Med. 2019;17(1):31.

7. Van Bavel JJ, Boggio P, Capraro V, Cichocka A, Cikara M, Crockett $\mathrm{M}$, et al. Using social and behavioural science to support COVID-19 pandemic response. Nat Hum Behav. 2020.
8. Chew QH, Wei KC, Vasoo S, Chua HC, Sim K. Narrative synthesis of psychological and coping responses towards emerging infectious disease outbreaks in the general population: practical considerations for the COVID-19 pandemic. Singap Med J. 2020.

9. Gaudine A, Gien L, Thuan TT, Dung DV. Perspectives of HIVrelated stigma in a community in Vietnam: a qualitative study. Int J Nurs. 2010;47(1):38-48

10. NCIRD. Reducing Stigma 2020 [Available from: https://www.cdc. gov/coronavirus/2019-ncov/daily-life-coping/reducing-stigma.html\# t1.

11. Korte KJ, Denckla CA, Ametaj AA, Koenen KC. At Harvard T. H. Chan. Stigma: Viruses Don't Discriminate and Neither Should We! 2020 [Available from: https://gsas.harvard.edu/student-life/harvardresources/stigma-viruses-dont-discriminate-and-neither-should-we.

12. UN. The Impact of COVID-19 on older persons. 2020.

13. lum M. Covid-19: Stop the stigma and stereotyping of coronavirus sufferers 2020 [Available from: https://www.thestar.com.my/lifestyle/ health/2020/03/17/fighting-against-the-stigma-of-covid-19.

14. Logie CH, Turan JM. How Do We Balance Tensions Between COVID-19 Public Health Responses and Stigma Mitigation? Learning from HIV Research. AIDS Behav. 2020:1-4.

15. WHO. Mental health and psychosocial considerations during the COVID-19 outbreak, 18 March 2020. World Health Organization; 2020.

16. Combating bias and stigma related to COVID-19 2020 [Available from: https://www.apa.org/topics/covid-19-bias.

17. O'Brien A. Covid 19. The paradox of social distancing. J Psychiatr Ment Hlt. 2020.

18. Guterres A. The recovery from the COVID-19 crisis must lead to a different economy: UN; 2020 [Available from: https://www.un.org/ en/un-coronavirus-communications-team/launch-report-socio-econom ic-impacts-covid-19. 